\title{
Using Petri Net Invariants in State Space Construction
}

\author{
Karsten Schmidt \\ Institut für Informatik, Humboldt-Universität zu Berlin, D-10099 Berlin \\ kschmidt@informatik.hu-berlin.de
}

\begin{abstract}
The linear algebraic invariant calculus is a powerful technique for the verification of Petri nets. Traditionally it is used for structural verification, i.e. for avoiding the explicit construction of a state space. In this paper, we study the use of Petri net invariants for reducing the memory resources required during state space construction. While place invariants help to reduce the amount of memory needed for each single state (without reducing the number of states as such), transition invariants can be used to reduce the number of states to be stored. Interestingly, our approach does not require computing invariants in full, let alone storing them permanently. All information we need can be deduced from an upper triangular form of the Petri net's incidence matrix. Experiments prove that the place invariant technique leads to improvements in both memory and run time costs while transition invariants lead to a space/time tradeoff that can be controlled heuristically.
\end{abstract}

\section{Introduction}

Petri net invariants are a well understood and broadly used concept in the verification of Petri nets. Place invariants can be used to closely over-approximate the state space thus being useful for verifying various safety properties. Transition invariants give hints to cycles, other than this they have been of some limited use in verification [13. So far, invariants have been mainly studied as structural analysis techniques, i.e. as a tool to replace explicit state space verification. In this paper, we propose methods to use them for improving state space verification. The general ideas are not very deep from a theoretical perspective, but turned out to lead to significant improvements in run time and space of explicit state space verification.

For place invariants, we use the fact that a place invariant corresponds to a linear equation that involves the markings of several places. Given such an equation, the marking of one of the places is therefore determined by the marking of the remaining places. Consequently, it is not necessary to store the marking of that particular place in the state space data structure. Given $n$ linearly independent place invariants, only $|P|-n$ places need to be stored to uniquely identify a state. Our experiments suggest that a vector to be stored can be shrunk to 50-70 percent of its original size. Since many state space related operations operate then on the shrunk vector, we obtain significant run time improvements 
that more than compensate the overhead for acquiring the necessary information about place invariants.

Techniques for compressing state representations based on place invariants have been proposed, for instance, in [14. There, they use specific place invariants that allow us to deduce that exactly one place in the support of the invariant contains exactly one token while the remaining places are empty. In such a situation (assuming $n$ places in the support of the invariant) they can shrink the $n$ places to a representation of size $\log n$. However, this approach requires full computation of particular invariants (which can be done by linear programming) while our approach is based on much simpler information about place invariants which can be computed even more efficiently.

For transition invariants, we use their capability of hinting at cycles in the state graph. Every transition sequence that can occur in some state and returns to the same state, corresponds to a transition invariant. Now it is easy to verify that for termination of reachability graph generation it is sufficient to store as many states as are necessary to cover all cycles of the graph (i.e. to have one state of the cycle stored). The other states will be generated over and over again (without storing them, thus without detecting that they have been generated before) but since these states do not form cycles they do not pose any threat for termination of reachability graph generation. We show that, given some knowledge about transition invariants, a set of states can be characterized that covers all cycles of the net. This number is reasonably small thus allowing for a significant state space reduction. Unfortunately, the repeated generation of states leads to an exponential blow up in the run time. This blow up is not as bad when partial order reduction is applied in connection with the transition invariant reduction (since partial order reduced graphs have smaller branching factors). However, we found it necessary to implement a configurable tradeoff between space and time. Given a user configurable number $k$, we store, in addition to the states required by the invariant method, all states that are encountered in a distance from the initial marking that is divisible by $k$. This way, the amount of repeated encounters of other states can be limited, for the price of more space costs.

Only few Petri net tools use a state compression based on place invariants (in fact, besides our own tool LoLA [20] we only know of INA [15] using this technique). The reason might be that most other Petri net tools have high level Petri nets as their base formalisms, in contrast to INA's and LoLA's low level

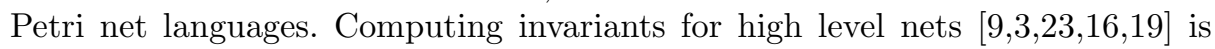
a much more involved task than computing low level net invariants where we "only" need to solve a system of linear equations with integer coefficients.

An approach similar to the transition invariant approach has been reported for the real time verification tool UPPAAL [12]. They verify systems that are parallel compositions of timed state machines and use depth first search on the component state machines for identifying cycles in the components. Those cycles are used to characterize a set of states covering all cycles in the reachability graph. 
As the actual contribution of this paper, we show that both our approaches do not even depend on the actual computation of invariants, let alone having the invariants explicitly stored in memory. All information we need can be deduced from transforming the matrix that defines the system of linear equations to be solved into an upper triangular form. On models with 10,000 Petri net nodes, this task does still take less than one second, so the preprocessing overhead can be neglected.

We first introduce Petri nets, related state spaces, and state equations. The latter concept is the starting point for both invariant calculi. We then sketch an algorithm for depth first state space generation and present the two reductions. We continue with a method that can deduce all information we need about invariants from upper triangular forms of the involved systems of equations. We conclude with some experimental results and a discussion on compatibility with other state space reduction techniques.

\section{Petri Nets, State Space, and State Equation}

Let $\mathbf{N}$ denote the set of natural numbers, including 0 . Consider a Petri net $N=\left[P, T, F, W, m_{0}\right]$ with a finite set $P$ of places, a finite set $T$ of transitions, a set $F \subseteq(P \times T) \cup(T \times P)$ of arcs, an arc weight function $W:(P \times T) \cup(T \times P) \longrightarrow \mathbf{N}$ such that $W([x, y])=0$ if and only if $[x, y] \notin F$, and a marking $m_{0}$, the initial marking. A marking is a mapping $m: P \longrightarrow \mathbf{N}$ which is usually interpreted as a distribution of tokens on the places, with $p$ carrying $m(p)$ tokens. A transition $t$ is interpreted as changing the distribution of tokens by removing $W([p, t])$ tokens from $p$ (for all places $p$ ), and producing $W([t, p]$ ) tokens on $p$ (for all places $p$ ). Thus, a marking $m^{\prime}$ is immediately reachable by a transition $t$ from a marking $m$ (written $\left.m \stackrel{t}{\rightarrow} m^{\prime}\right)$ iff, for all $p, m(p) \geq W([p, t])$, and $m^{\prime}(p)=$ $m(p)-W([p, t])+W([t, p])$.

The Petri net depicted in Fig. 1 models the well known dining philsophers example. In the picture, places are represented by circles, transitions by rectangles, arcs as arrows. In the example, weights are 0 (no arrow) or 1 (arrow present). The initial marking is represented by black dots on places.

Reachability can be extended to sequences of transitions by letting $m \stackrel{\varepsilon}{\rightarrow} m$ for all markings $m$ and the empty sequence $\varepsilon$, and $m \stackrel{w t}{\longrightarrow} m^{\prime}$ iff there is a marking $m_{1}$ such that $m \stackrel{w}{\longrightarrow} m_{1}$ and $m_{1} \stackrel{t}{\rightarrow} m^{\prime}$ (with $w$ being a sequence of transitions and $t$ a transition). $m^{\prime}$ is just reachable from $m\left(m \stackrel{*}{\rightarrow} m^{\prime}\right)$ iff there is a transition sequence $w$ such that $m \stackrel{w}{\longrightarrow} m^{\prime}$.

The state space of a Petri net consists of the set $R_{N}\left(m_{0}\right)=\left\{m \mid m_{0} \stackrel{*}{\rightarrow} m\right\}$ of states reachable from the initial marking. It can be extended to a graph by letting $R_{N}\left(m_{0}\right)$ be the vertices and . $\stackrel{t}{\rightarrow}$. be the edges labeled with transitions. Most properties of interest are defined, and can be verified, as patterns of the state graph. Therefore, we present our technique, without discussing particular properties, using an algorithm that just generates the state graph. 


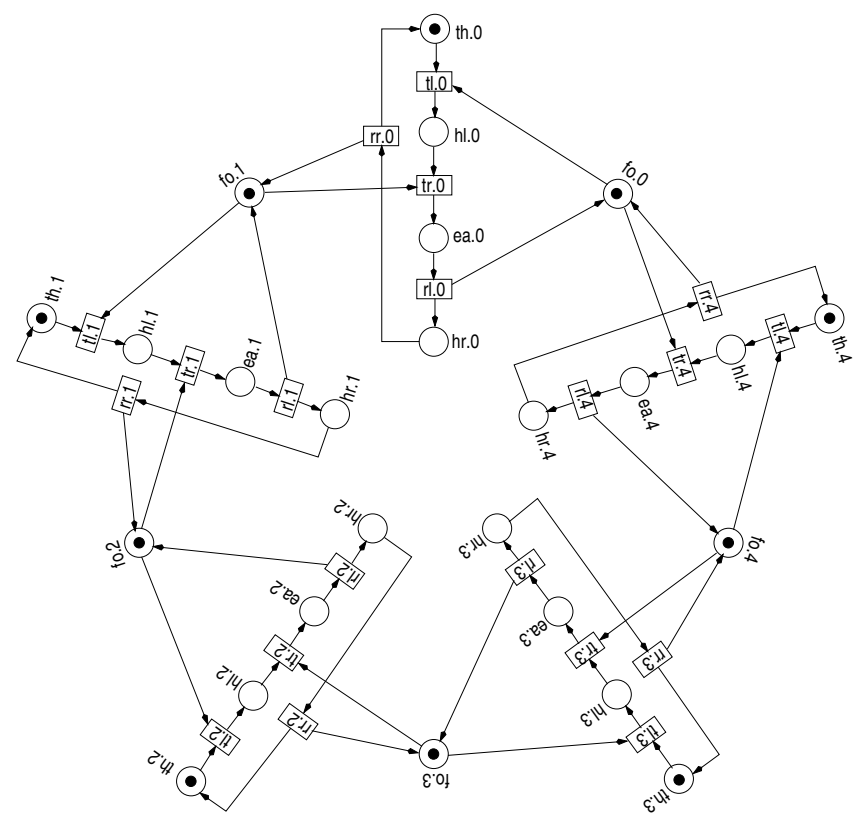

Fig. 1. This Petri net models the 5 dining philosophers system. The internal state of philospher $i$ is marked by a token on th. $i$ (thinking), hl. $i$ (has left fork), ea. $i$ (eating), or hr. $i$ (has right fork). A token on fo. $i$ models an available fork. Transitions shift states by removing the token representing the current state, producing a token representing the next state, and consuming or producing (or: taking or releasing) a fork.

The incidence matrix $C$ of a Petri net $N$ has a row for each place $p \in P$ and a column for each transition $t \in T$. It is defined by $C(p, t)=W(t, p)-W(p, t)$.

From the definition of reachability, it follows that $m \stackrel{w}{\longrightarrow} m^{\prime}$ (for a sequence w) implies

$$
m^{\prime}=m+C \cdot \Psi(w)
$$

where $\Psi(w)$ (the count vector of $w$ ) is a column indexed by $T$ where $\Psi(w)(t)$ corresponds to the number of occurrences of $t$ in $w$, and markings are considered as $P$-indexed columns. This equation is called the Petri net state equation. Throughout this paper, $A \cdot B$ denotes the inner product between two matrices $A: I_{1} \times I_{2} \longrightarrow \mathbb{Q}$ and $B: I_{2} \times I_{3} \longrightarrow \mathbb{Q}$ (including vectors as matrices with 1 as one of their dimensions), and $A \cdot B(i, k)=\sum_{j \in I_{2}} A(i, j) \cdot B(j, k)$ for all $i \in I_{1}$ and $k \in I_{3}$.

\section{Invariants}

A place invariant is an integer row vector $i$ such that $i \cdot C=\underline{0}$ where $\underline{0}$ is a null vector of appropriate dimension, and $i$ has $P$ as its index set. The requirement of having an integer vector can be relaxed to rational, or real numbers without 
Table 1. Depth first generation of state graph for $N=\left[P, T, F, W, m_{0}\right] ; t^{-}$and $t^{+}$are $P$-indexed vectors with $t^{-}(p)=W([p, t])$ and $t^{+}(p)=W([t, p])$

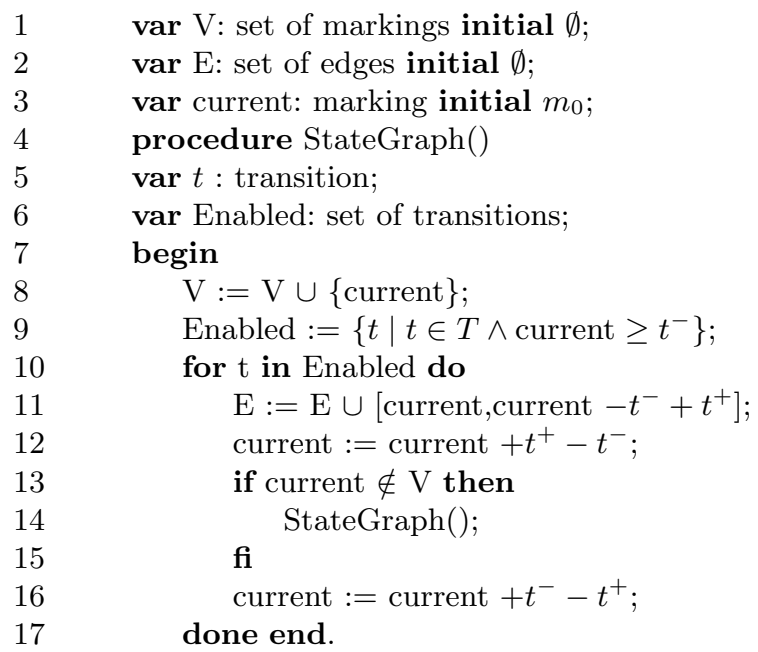

significant impact on our approach. The main property of an invariant $i$ is that, for a marking $m^{\prime}$ reachable from another marking $m$, it holds that $i \cdot m^{\prime}=i \cdot m$. Thus, every reachable state has the same product with $i$ as the initial marking. The main property can be easily verified by multiplying $i$ from the left to the state equation.

One example for a place invariant in Fig. 1 is the vector assigning 1 to places fo.0, hl.0, ea.0, ea.4, and hr.4 while assigning 0 to the remaining places.

A transition invariant is an integer column vector $i$ such that $C \cdot i=\underline{0}$ where $i$ has $T$ as its index set. It follows directly from the state equation that for every transition sequence $w$ where $m \stackrel{w}{\longrightarrow} m$ for some $m$ (forms a cycle), $\Psi(w)$ is a transition invariant.

One example for a transition invariant in Fig. 1 is the vector assigning 1 to transitions tl.0, tr.0, rl.0, and rr.0 while assigning 0 to all remaining transitions.

\section{Place Invariant Based State Compression}

Let $i$ be a place invariant and $p$ be a place such that $i(p) \neq 0$. Let $m$ be a marking. From the main property of place invariants, we have

$$
m(p)=\frac{i \cdot m_{0}-\sum_{p^{\prime} \in P \backslash\{p\}} i\left(p^{\prime}\right) \cdot m\left(p^{\prime}\right)}{i(p)}
$$

Thus, $m(p)$ can be completely determined from $m$ on the remaining places, $i$, and the (constant) initial marking $m_{0}$. Consequently, it is not necessary to store $m(p)$ explicitly for any reachable marking $m$. Using the place invariant mentioned in 
the previous section, we can, in every reachable marking, deduce the token count in fo. 0 from the token counts on hl.0, ea.0, ea.4, and hr.4: if any of those places contains a token then fo.0 does not, otherwise it contains exactly one token.

A net usually has more than one place invariant. Consequently, $n$ linearly independent invariants can be used to exempt $n$ components of a marking from explicit storage.

Assume that $P$ is partitioned into a set $S$ of significant places and $R$ of redundant places $(P=S \cup R, S \cap R=\emptyset)$ such that for each $p \in R$ there is a place invariant $i_{p}$ holding $i_{p}(p) \neq 0$ and $i_{p}\left(p^{\prime}\right)=0$ for $p^{\prime} \in R \backslash\{p\}$. This means, according to previous considerations, the marking on all redundant places can be reconstructed from the marking on significant places. Notice that $\left\{i_{p} \mid p \in R\right\}$ is a linearly independent set of invariants. Furthermore, due to the ability of uniquely reconstructing the whole vector, we have that for two reachable markings $m$ and $m^{\prime}, m=m^{\prime}$ if and only if $m(p)=m^{\prime}(p)$ for all $p \in S$.

In other words, the portion of $m$ that corresponds to $S$ uniquely identifies a reachable state. This comparison is the only operation that is ever done with states other than the current state. A further look at the depth first search algorithm in Tab. 1 shows that the current state itself is never loaded from the set $V$ of known states but always computed from firing transitions forward or backward 1 This is an efficient solution anyway since firing a transition (forward or backward) involves only places in the immediate $F$-neighborhood of the transition while copying a state involves the whole $P$.

Thus, assuming that we only store the $S$-portion of $m$ in the set of known states, we can preserve the full functionality of the sketched depth first search. All we need to know is a set $S$ of significant places satisfying the above requirements, the actual vectors $i_{p}$ are completely irrelevant (beyond the determination of $S$ ). Search and insert operations to the set $V$ now involve only $S$-vectors rather than $P$-vectors which speeds up both operations. Our experiments show that the additional run time to determine $S$ more than pays off through these speed ups.

The actual determination of $S$ and $R$ is left to Sec. 6 .

In the model in Fig. 1 one possible partition has all places hl.i, ea. $i$, and hr. $i$ as significant places, all remaining places are redundant. Thus, the size of every state vector shrinks from 25 components to 15 components.

Actually, there are several possibilities to partition $P$. Correctness of our approach is independent of the particular choice. The maximum number of elements in $R$ is equal to the number of linearly independent place invariants in the system. The construction below does always yield such a maximum size set $R$.

\footnotetext{
${ }^{1}$ Backward firing refers to step 16 of the algorithm presented in Sec. 3. This is the step where we backtrack from a completely processed state to one of its predecessor state during (forward) reachability analysis. This step should not be confused with backward reachability analysis.
} 


\section{Transition Invariant Based Reduction}

As already sketched in the introduction, we aim at identifying a set $Z \subseteq R_{N}\left(m_{0}\right)$ of states such that every cycle in the reachability graph contains at least one element of $Z$. Transition invariants characterize count vectors of transition sequences that corresponds to cycles: if a transition sequence returns to the start state, its count vector is a transition invariant.

In Fig. 1, the sequence tl.1, tr.1, rl.1, rr.1 can be executed in the initial state and reproduces the initial state. The vector assigning 1 to the mentioned transitions and 0 to the remaining transitions is a transition invariant.

Consider a set $U$ of transitions such that every transition invariant (other than the vector of all zeros) is non-zero for at least one element in $U$. Then, consequently, every cycle in the state graph contains a state $m$ where an edge labeled with an element $t$ of $U$ springs off, i.e. $t$ is enabled at $m$. Thus, the set of states where at least one element of $U$ is enabled satisfies the condition posed on $Z$ above.

Applying transition invariant based reduction means that states that enable elements of $U$ are stored in the set $V$ of known states while other states are computed and explored, but not stored permanently. Given a set $U$, determining whether or not a marking $m$ enables an element of $U$ can be embedded efficiently into the calculation of enabled transitions at $m$. If we explore, for a second time, a state that does not enable a transition in $U$, we do not find this state in $V$, so we compute its successors a second time. However, since ignored states do not form complete cycles, termination of reachability graph generation is still guaranteed.

In preprocessing, only $U$ needs to be determined while, as in the case of place invariants, it is not necessary to know the actual invariants.

While the place invariant based technique leads to gains in both space and time efficiency, the transition invariant based technique can become extremely slow, since states that are not stored permanently can be visited and explored a large (up to exponential) number of times. This problem is less grave when the transition invariant technique is combined with partial order reduction. Partial order reduction narrows the number of successors to be computed in each state. Thus, the average number of predecessors of a states shrinks as well, and that number determines how often not permanently stored states would be reexplored. Still, first experiments suggested that it is necessary to further improve the time efficiency of the method. We decided to gain time efficiency by storing more states permanently (thus re-exploring less states). We experimented with a criterion that stores states if they are explored in a distance that is divisible by some user specified number $k$. Thus, regarding the depth first search tree, certain layers of the tree are stored completely, in addition to the states that potentially close cycles. In this combination, the choice of $k$ controls a space/time tradeoff where large values mean trading time for getting better space efficiency while small numbers increase time efficiency while requiring more space.

With not saving all states, it does not make sense to store edges. Therefore the sketch of the transition invariant based reduction in Tab. 20 does not contain 
edges at all. Nevertheless every state will be eventually visited by the algorithm. Thus, simple safety properties such as reachability, deadlocks, etc., can still be exhaustively verified.

Table 2. Depth first generation of state graph with transition invariant based reduction for $N=\left[P, T, F, W, m_{0}\right] ; t^{-}$and $t^{+}$are $P$-indexed vectors with $t^{-}(p)=W([p, t])$ and $t^{+}(p)=W([t, p])$; let $k$ be a nonzero natural number and $U$ as described in the text

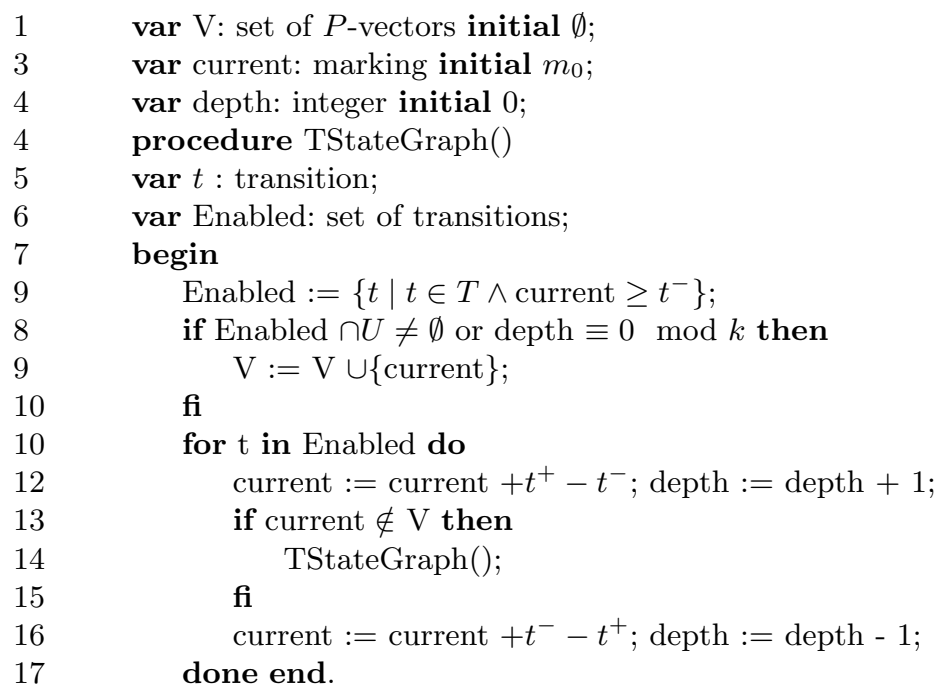

\section{Upper Triangular Form}

In this section, we demonstrate how efficiently a partition of $P$ into sets $S$ of significant and $R$ of redundant places can be computed based on well known linear algebra. Determining a set $U$ of transitions needed for the transition invariant technique is equally simple.

Consider a homogeneous system $A \cdot x=\underline{0}$ of linear equations ( $A$ can be the incidence matrix $C$ for transition invariant, or the transposed of $C$ for place invariant calculation). It is well known that exchanging rows in $A$, adding a row of $A$ to another, or multiplying a row by a scalar, are operations that preserve the space of solutions and can be used to transform the matrix into an upper triangular form, that is into a form where the leftmost nonzero element of a row is always right of the leftmost nonzero element of the previous row. Call the columns that contain the leftmost nonzero element of some row head columns, and call columns that do not contain a leftmost nonzero element of any row tail columns. 
Example. The matrix

$$
\left(\begin{array}{lllllll}
1 & 0 & 1 & 1 & 0 & 2 & 3 \\
0 & 1 & 1 & 2 & 2 & 1 & 1 \\
0 & 0 & 0 & 0 & 2 & 1 & 0 \\
0 & 0 & 0 & 0 & 0 & 0 & 1
\end{array}\right)
$$

is in upper triangular form. Columns 1, 2, 5, and 7 are head columns while columns 3,4 , and 6 are tail columns.

Columns of $A$ correspond to variables in $x$. Thus, we can partition variables correspondingly into head variables and tail variables. In the example, we would have head variables $x_{1}, x_{2}, x_{5}$, and $x_{7}$, and tail variables $x_{3}, x_{4}$, and $x_{6}$. The partition into head and tail variables depends on the particular upper triangular form, especially on the order of indices in the index sets of the involved matrix. The number of head and tail variables is, however, determined by the rank of the matrix.

Now, consider a tail variable $x_{j}$, a partial assignment to $x$ where $x_{j}=1$, and $x_{k}=0$ for all remaining tail variables. We can easily see that every such partial assignment can be extended to a solution of the whole system of equations. For this purpose, start at the bottom row of $A$. This row has, besides some of the tail columns, only its own head column as nonzero entry. Thus, the partial assignment can be extended, by assigning some rational value (zero or nonzero), to a solution for the bottom equation of the upper triangular form. Values of other head variables are irrelevant since they have zero coefficients in the bottom equation. Now, going up step by step, we have always a partial assignment to all variables that have nonzero entries, except the head variable of the considered row, and an appropriate assignment to that variable solves the new equation without interfering with equations below. Consequently, every partial solution that assigns 1 to some tail variable and 0 to the remaining tail variables, can be extended to a unique rational solution (and, by multiplying by a common divisor, to an integer solution) of the system of equations that is unique up to multiplying all components by a scalar. Rank considerations show, that the set of solutions obtained this way forms a basis of the whole solution space (the number of head variables corresponds to the rank of $A$, the number of all variables to the dimension of $x$ ).

Without knowing the values of solutions on the head variables, we have sufficient information for both invariant based state space techniques. For the place invariant approach, we need to partition $P$ into significant and redundant places. Since places correspond to variables in the system of equations corresponding to place invariants, we can let $S$ be the head variables of the upper triangular form, and $R$ be the tail variables. This setting matches exactly the definition of significant and redundant places.

For transition invariants, we can observe that there cannot be any nonzero transition invariant that assigns 0 to all tail variables. Since the set of invariants that can be obtained by the above procedure forms a basis for the set of all solutions to the system of equations (the solutions are obviously linearly independent), a solution that assigns 0 to all tail variables must be representable as 
a linear combination of the base vectors. By construction, only the zero vector can be a linear combination of the described solutions that assigns 0 to all tail variables. Thus, the set $U$ of all transitions that correspond to tail variables in the system of equations has the property that every nonzero transition invariant has a nonzero element in $U$. Moreover, this set is minimal at least with respect to set inclusion, since for every proper subset of this $U$, one of the generators we described would have all its nonzero entries outside $U$.

In conclusion, gaining the knowledge needed for our techniques requires less effort than even computing the actual invariants!

\section{Compatibility with Other Reduction Techniques}

Place invariant based compression is compatible with most other frequently used reduction techniques. For all known partial order reduction techniques (see [24] for a survey), it is sufficient to investigate the current state. For some advanced techniques, the state graph as such must be investigated for strongly connected components and transitions occurring in strongly connected components. None of this information is altered by our compression technique based on place invariants.

For symmetry reduction $7 / 228,24110,17$ 18], the test whether the current state is in $V$ must be replaced by a test whether there is a symmetry such that the symmetric image of the current state is in $V$. There are different solutions to this problem, 18 lists several of them. One solution is to iterate all symmetries, apply a symmetry to the current state and check for containment of the resulting symmetric image of the state. In this solution, the projection of the current state to $S$ can be done after the application of the symmetry, so there is no compatibility problem. A second solution uses symmetries to transform the current state into a canonical representative of its class and checks whether the representative is in $V$. Again, there is no problem since the projection to $S$ can be applied to the representative. A third solution proposed in [21] proposes to iterate through $V$ (more precisely, the subset of $V$ that matches the hash value of the current state), and tries to calculate a symmetry from the iterated states to the current state. This approach would require restoring elements of $V$ to full markings which is not covered by our approach. Thus, symmetry reduction can be used in connection with place invariant compression, but not all of the existing methods for checking containment of a symmetric image can be applied.

Coverability graph generation [11,5] involves checking whether the current state covers any predecessor state on the path back to the initial state. If so, the coverability graph algorithm assigns the value $\omega$ to every properly covering component. In order to be compatible with place invariant based reduction, we can store information about $\omega$-introductions separated from the actual marking vectors. So, we can attach to each state a list of places where a $\omega$ is introduced exactly at that state, together with the old (finite) value that is replaced with $\omega$. This way, we can generate all states on the path from the initial state to the current state, by starting with the current state, firing transitions backwards 
in connection with updating a pointer to the corresponding $S$-vector in $V$. If that pointer points to a state with attached information about $\omega$-introductions, that information can be used to restore the value of the vector before introduction of the new $\omega$. This way, all states on the path from the initial state to the current state can be generated without actually retrieving states from $V$. Since two subsequent states differ in only few components (the environment of the fired transition), this method of iterating states is very efficient anyway. In conclusion, place invariant based state compression is compatible with coverability graph generation. This result holds, however, only for the original coverability graph algorithm proposed in 11. For the minimal coverability graph algorithm proposed in [5], not only states on the path back to the initial state, but all states in $V$ are checked for covering. For this covering check it is neither sufficient to use only the $S$-projection of states in $V$, nor is there an efficient way to restore those states without knowing the actual values of place invariants.

There are other compression techniques than place invariants. Most of them [6 25] rely on hash functions on the states. Applying these techniques to the $S$ projection of a marking instead of the full marking can speed up the calculation of hash values. Moreover, since the components removed by the place invariant compression are functionally dependent on the remaining components, applying hash techniques to the compressed vector can even lead to a more perfect (i.e. more uniformly spreading) behavior of the hash function based techniques. So far, we have not verified this conjecture experimentally.

For symbolic state spaces, for instance, based on BDDs, the compression might be of little value. Reducing the BDD to $S$ is no solution since we need the full state in order to compute enabled transitions and successor markings. In explicit state space analysis, this is fully covered by having the current state available in uncompressed form. In symbolic state space analysis, enabledness and successors are computed from the BDD, so the reduced information is insufficient. Remember that an important pre-requisite of our technique is to restore current states by firing transitions forward or backward, rather than copying them from $V$.

Since the transition invariant based technique only controls which markings are permanently stored, but not which successors are explored at a marking, there are no compatibility problems with basic partial order reduction techniques. With basic partial order reduction methods we mean techniques where the determination of the set of transitions to be fired at a state can be determined from the current state and the net structure. The original deadlock preserving stubborn set technique, and several of the stubborn set approaches for Petri net standard properties are in this class of partial order reduction methods. For advanced techniques (such that rely on investigations on connected components of the state graph, for instance the LTL preserving and CTL* preserving methods; refer to the survey [24] for details), we need some future research to check whether the necessary information can be retrieved from the reduced graph (the original edge relation is unavailable due to the removal of states). Basic partial order reduction techniques, however, increase the performance of the transition 
invariant technique by reducing the average branching factor in the state graph. Cycles in a graph reduced by partial order reduction, are definitely cycles in the original graph, so the transition invariant technique works correctly for partial order reduced graphs (in the sense that reduced graph generation terminates).

For symmetries, we need to be careful since cycles in a symmetrically reduced graph do not necessarily correspond to cycles in the original graph. The reason is that a marking can have an edge to a symmetric image of its actual successor marking. However, a cycle in the reduced graph can be extended to a cycle in the original graph since for every sequence executable in a state, a sequence consisting of equivalent transitions can be executed at a symmetric state. Thus, for every cycle in the reduced graph there is a cycle in the original graph (maybe longer) that consists of the transitions occurring in the reduced graph's cycle and transitions equivalent to them. Therefore, enlarging $U$ by all transitions that are equivalent w.r.t. symmetry to elements in $U$ can guarantee that the enlarged $U$ contains at least one transition for each cycle in the symmetrically reduced state graph.

Transition invariant based reduction and coverability graph reduction according to 11] are compatible when the technique described earlier is used to generate all states from the current state back to the initial state.

Application of hash function based compression techniques does not interfere with the transition invariant based reduction; a combination with BDD based techniques seems not to be feasible.

\section{Experiments}

As examples, we use a sequence of $n$ dining philosophers examples, and a sequence of a semaphore based scheme for concurrent read and exclusive write access to a data base for $n$ writing and $n$ reading processes.

An $n$ dining philosophers system, in the version we use, has $5 n$ places and $2 n$ linearly independent place invariants. Thus, the reduction in terms of vector length is $40 \%$. The data base scheme with $n$ reading and $n$ writing processes has $6 n+1$ places and $3 n+1$ independent place invariants. Thus, the vector length is reduced to approximately $50 \%$. In several other examples, we got reductions from $30 \%$ to $50 \%$. In run time (all numbers are measured on one and the same computer and include the whole task including reading the net, processing invariants (for reduced version), and generating the full state graph), we got the following numbers?.

The reported systems are tiny since we wanted to compare run times between place invariant reduction and brute force state space generation. In connection with other reduction techniques, the speed up is comparable. For instance, verifying a 500 philosophers system (with an overall of $3^{500}-1$ states, the partial

\footnotetext{
${ }^{2}$ All reported run times relate to the implementation of both methods in the tool LoLA 20, running on a LINUX machine equipped with a $650 \mathrm{MHz}$ Pentium processor and 256 MByte of RAM.
} 
Table 3. Speedup introduced by place invariant compression.

\begin{tabular}{lcccccc}
\hline & 10 phil 11 phil 12 phil 14 data & 16 data & 18 data \\
\hline states & 59048 & 117146 & 531440 & 16398 & 65552 & 262162 \\
time (sec) without red. & 2.7 & 9.8 & 37.7 & 2.0 & 10.4 & 53.8 \\
time (sec) with red. & 2.1 & 7.7 & 30.3 & 1.3 & 6.7 & 35.8 \\
\hline
\end{tabular}

order reduced state space consists of 751502 states. It takes $149 \mathrm{sec}$. to compute it with place invariant compression, compared to 238 sec. without. Using both partial order and symmetry reduction on the 1000 dining philosophers system, the reduced state space consists of 2997 states (compared to $3^{1000}$ states of the full state space). Computation requires $189.4 \mathrm{sec}$. with place invariants involved, compared to 189.7 without. In this case, numbers are almost equal since most of the time is spent for investigating symmetries.

For the transition invariant technique, it is interesting to study the impact of the heuristical parameter $k$ that controls the amount of additionally stored states. An $n$ philosophers system has $n$ independent transition invariants. With $n$ writing and $n$ reading processes, the data base scheme has $2 n$ independent transition invariants.

The first two tables show the limited capabilities of transition invariant based reduction without combining it with partial order reduction. The first table shows the impact of $k$. For the data base example, larger values of $k$ do not change the number of states which can be blamed to very tight cycles in that net.

Table 4. State reduction and time overhead in transition invariant reduction on the dining philosophers system.

\begin{tabular}{lccccc}
\hline & 5 phil 6 phil 7 phil 8 phil 9 phil \\
\hline states without red. & 242 & 728 & 2186 & 6560 & 19682 \\
time (sec) without red. & 0.03 & 0.04 & 0.08 & 0.23 & 0.76 \\
states with red., $k=5000$ & 160 & 530 & 1708 & 5417 & 16952 \\
time (sec) with red., $k=5000$ & 0.09 & 0.7 & 9.7 & 136.0 & 2177.6 \\
states with red., $k=20$ & 186 & 591 & 1828 & 5664 & 17545 \\
time (sec) with red., $k=20$ & 0.05 & 0.1 & 0.36 & 3.19 & 10.8 \\
states with red., $k=10$ & 201 & 629 & 1947 & 5984 & 18289 \\
time (sec) with red., $k=10$ & 0.04 & 0.07 & 0.19 & 0.59 & 1.8 \\
\hline
\end{tabular}

The last table shows the behavior of transition invariant based reduction in connection with partial order reduction. We use deadlock preserving stubborn sets as the partial order reduction technique. The numbers show a significantly better performance of the transition invariance technique. 
Table 5. State reduction and time overhead in transition invariant reduction on the data base system.

\begin{tabular}{lccc}
\hline & 14 data & 16 data 18 data \\
\hline states & 16398 & 65552 & 262162 \\
time (sec) without red. & 2.0 & 10.4 & 53.8 \\
states with red. $k=10$ & 16384 & 65536 & 262144 \\
time (sec.) with red. $k=10$ & 2.1 & 10.4 & 55.1 \\
\hline
\end{tabular}

Table 6. Combination of transition invariant reduction with partial order reduction.

\begin{tabular}{lccccc}
\hline & 100 phil & 200 phil & 200 data & 300 data 400 data \\
\hline states (p.o. red. only) & 29702 & 119402 & 401 & 601 & 801 \\
time (sec) (p.o. red. only) & 2.2 & 16.4 & 8.2 & 25.1 & 61.0 \\
states both red., $k=5000$ & 10311 & 41093 & 1 & 1 & 1 \\
time (sec.) both red., $k=5000$ & 45.3 & 395.3 & 13.0 & 26.8 & 64.6 \\
states both red., $k=20$ & 14502 & 59002 & 1 & 1 & 1 \\
time (sec.) both red., $k=20$ & 3.5 & 26.5 & 8.0 & 26.7 & 64.4 \\
states both red., $k=10$ & 17702 & 71402 & 1 & 1 & 1 \\
time (sec.) both red., $k=10$ & 2.8 & 21.4 & 8.3 & 26.7 & 64.0 \\
\hline
\end{tabular}

\section{Discussion}

The place invariant technique yields improvements in both space and time. It is compatible with many other reduction techniques. Thus, no special care needs to be taken when applying the method. The transition invariant based approach turns out to be unfeasible as a standalone technique but can be rather valuable when applied in connection with partial order reduction. It requires that the parameter $k$ that controls the space/time tradeoff of the technique be chosen carefully. Then, however, it can make the difference between getting a state graph into the available memory or not.

Feasibility of both methods relies on the fact that we do not need the actual values of the invariants but only information that, in a generating set, certain elements are guaranteed to be 0 or nonzero. This way, no space is required for storing invariants, and preprocessing can be reduced to just generating an upper triangular form out of the net's incidence matrix (or its transposed).

Besides the fact that the linear algebraic approach to invariance is already rather Petri net specific (even low level net specific), our approach relies essentially on the Petri net specific fact that we can backtrack from a successor state by firing a transition backwards. Many other system description languages are based on programming language like notations where constant assignments (for example, $x:=0$ ) cannot be applied backwards that easily. In such a situation, 
it would be more difficult to live without the ability to re-construct complete states.

For high level nets, several obstacles make the place invariant technique unfeasible. First, an automatic calculation of place invariants is a computationally involved task. The approach would therefore need to rely on user supplied invariants. Second, the supplied invariants must enable the system to deduce a partition into significant and redundant place instances (colours). Finally, many high level net tools compute successor states by executing programming language style code attached to arcs or transitions. In that framework, it is usually impossible to backtrack to a predecessor marking other than by restoring it from the depository of known states since the attached code cannot be executed "backwards".

In contrast, the transition invariant technique can be adapted to high level nets without major problems, at least when the high level net formalism is such that all bindings of a high level net transition cause the same number of tokens to be produced or consumed at every pre- and post-place of the transition. In that case, the skeleton of the high level net (replacing all coloured tokens by black tokens) is well defined and every cycle in the high level net state space corresponds to a cycle in its skeleton. Then, computing $U$ in the skeleton, and storing all states of the high level net that enable at least one binding of some transition in $U$ solves the problem connected with our transition invariant based reduction method.

\section{References}

1. G. Chiola and G. Franceschinis. Colored gspn models and automatic symmetry detection. In Proceedings of the 3rd Int. Workshop on Petri Nets and Performance Models 1989, Kyoto, Japan - Los Alamitos, CA, USA, pages 50-60. IEEE Computer Society Press, 1990.

2. E.M. Clarke, R. Enders, T. Filkorn, and S. Jha. Exploiting symmetry in temporal logic model checking. Formal Methods in System Design, 9:77-104, 1996.

3. J.M. Couvreur. The general computations of flows for coloured nets. Proc. of the 11th int. Workshop on Application and Theory of Petri nets, 1990.

4. E.A. Emerson and A. P. Sistla. Symmetry and model checking. Formal Methods in System Design, 9:105-131, 1996.

5. A. Finkel. A minimal coverability graph for petri nets. Proc. of the 11th International Conference on Application and Theory of Petri nets, pages 1-21, 1990.

6. G. Holzmann. On limits and possibilities of automated protocol analysis. Proc. 7th IFIP WG 6.1 Int. Workshop on Protocol Specification, Testing, and Verification, pages 137-161, 1987.

7. Huber, A. Jensen, Jepsen, and K. Jensen. Towards reachability trees for high-level petri nets. In Advances in Petri Nets 1984, Lecture Notes on Computer Science 188, pages 215-233, 1984.

8. C. Norris Ip and D. L. Dill. Better verification through symmetry. Formal Methods in System Design, 9:41-75, 1996.

9. K. Jensen. How to find invariants for coloured petri nets. Lecture Notes In Computer Science, 118:327-338, 1981. 
10. K. Jensen. Condensed state spaces for symmetrical coloured petri nets. Formal Methods in System Design, 9:7-40, 1996.

11. R. M. Karp and R. E. Miller. Parallel programm schemata. Journ. Computer and System Sciences 4, pages 147-195, Mai 1969.

12. K. Larsen, F. Larsen, P. Pettersen, and W. Yi. Efficient verification of real-time systems: compact data structure and state-space reduction. Proc. 18th IEEE RealTime Systems Symp., LNCS, pages 14-24, 1997.

13. K. Lautenbach and H. Ridder. Liveness in bounded petri nets which are covered by t-invariants. Proc. of the 15th international Conference on Theory and Application of Petri nets, Zaragoza, LNCS 815, pages 358-375, 1994.

14. E. Pastor and J. Cortadella. Efficient encoding schemes for symbolic analysis of petri nets. Proc. Proc. DATE '98, pages 790-795, 1998.

15. S. Roch and P. Starke. INA - Integrierter Netz-Analysator Version 1.7. Handbuch. Humboldt-University Berlin, Institute of Computer Science, 1997.

16. K. Schmidt. On the computation of place invariants for algebraic petri nets. Proceedings of the STRICT workshop Berlin, 1995.

17. K. Schmidt. How to calculate symmetries of petri nets. Acta Informatica 36, pages 545-590, 2000.

18. K. Schmidt. Integrating low level symmetries into reachability analysis. Proc. of the 6th International Conference Tools and Algorithms for the Construction and Analysis of Systems, LNCS 1785, pages 315-331, 2000.

19. K. Schmidt. T-invariants of algebraic petri nets. Informatik-Bericht, 31, 1994.

20. K. Schmidt. Lola: A low level analyser. Proc. Int. Conf. Application and Theory of Petri net, LNCS, 1825:465-474, 1999.

21. K. Schmidt. Integrating low level symmetries into reachability analysis. Proc. TACAS 2000, LNCS, 1785:315-331, 2000.

22. P. Starke. Reachability analysis of petri nets using symmetries. J. Syst. Anal. Model. Simul., 8:294-303, 1991.

23. J. Toksvig. Design and implementation of a place invariant tool for coloured petri nets. Master's thesis, University of Aarhus, 1995.

24. A. Valmari. The state explosion problem. In Lectures on Petri Nets I-Basic Models, LNCS 1491, pages 429-528, 1998.

25. P. Wolper and D. Leroy. Reliable hashing without collision detection. Proc. CAV, LNCS, pages 59-70, 1993. 